\title{
Reversible Hypokalemia and Bartter-Like Syndrome during Prolonged Systemic Therapy with Colistimethate Sodium in an Adult Patient
}

\author{
Tarek Kamal Eldin $^{1}$ (1) $\cdot$ Grazia Tosone $^{1} \cdot$ Alfredo Capuano $^{2} \cdot$ Raffaele Orlando $^{1}$
}

Published online: 11 July 2017

(c) The Author(s) 2017. This article is an open access publication

\begin{abstract}
We present the case of a 58-year-old woman who developed hypokalaemia and metabolic alkalosis 2 weeks after therapy with colistimethate sodium for the treatment of chronic lower limb ulcer infection by extensively drug-resistant (XDR) Pseudomonas aeruginosa. The metabolic changes observed resembled Bartter syndrome, a group of congenital disorders affecting the distal segments of the renal tubules. The metabolic abnormalities reversed spontaneously 6 days after drug discontinuation. Acquired forms of Bartter syndrome have been reported during courses of antibiotic therapy; however, to our knowledge, this is the first documented case associated with colistimethate therapy in an adult.
\end{abstract}

Tarek Kamal Eldin

tarek.kamaleldin@unina.it

1 Section of Viral Diseases, Department of Clinical Medicine, University Hospital 'Federico II' of Naples, Via Sergio Pansini, 5, 80131 Naples, Italy

2 Section of Haemodialysis Techniques and Peritoneal Dialysis, Department of Specialty Surgery and Nephrology, University Hospital 'Federico II' of Naples, Via Sergio Pansini, 5, 80131 Naples, Italy

\section{Key Points}

Patients receiving colistimethate sodium may present with hypokalaemia and electrolyte abnormalities suggestive of Bartter syndrome. Therefore, serum electrolytes must be monitored closely throughout the treatment course.

Electrolytes abnormalities during therapy with colistimethate may occur with normal renal function.

Electrolytes abnormalities during therapy with colistimethate appear to be reversible on interruption of treatment.

\section{Introduction}

Colistimethate is an old antibiotic that exerts bactericidal activity by disrupting bacterial cell membrane. It is active on Gram-negative organisms, including Pseudomonas aeruginosa [1]. Systemic use of colistimethate was initially discontinued because it is nephrotoxic. Colistimethate may cause dose-dependent acute kidney injury that is usually reversible and may require haemodialysis [2]. However, little is known about colistimethate-induced renal tubular damage. After multidrug-resistant (MDR) organisms emerged, the use of colistimethate was resumed, and it is now considered by many authors to be the salvage therapy of the twenty-first century [2]. With the emergence of healthcare-associated infections, infection by MDR organisms is no longer restricted to critically ill patients. The risk-benefit evaluation of antimicrobial treatment is 
therefore more challenging and requires extensive knowledge of the adverse effects of antimicrobial agents.

\section{Case Report}

We describe the case of a 58-year-old Caucasian woman who was admitted to our hospital for the management of chronic infected ulcers. She was a heavy smoker, was being treated with an angiotensin-converting enzyme (ACE) inhibitor (ramipril) for hypertension, and had chronic venous insufficiency. She reported undergoing surgical debridement of the ulcers and taking different antibiotics before admission. The ulcers were extensive and very painful. They involved most of the anterior aspect of both legs and were foul-smelling, shallow with non-healing punched edges. The wound bed showed extensive granulation tissue and necrotic slough. Biochemical exams and thyroid profile were within the normal range.

Because the patient refused a wound biopsy, culture and sensitivity testing was performed on a wound swab from which an extensively drug-resistant (XDR) [3] $P$. aeruginosa was isolated. Consequently, we started intravenous therapy with colistimethate sodium at a loading dose of 9 million units and a maintenance dose of 4.5 million units every $12 \mathrm{~h}$, meropenem $2 \mathrm{~g}$ every $8 \mathrm{~h}$, and gentamicin $80 \mathrm{mg}$ every $12 \mathrm{~h}$. We administered buprenorphine, pregabalin and paracetamol for pain control.

A few days later, we observed normalization of arterial blood pressure levels and, as expected, the development of non-oliguric stage 2 acute kidney injury according to KDIGO (Kidney Disease: Improving Global Outcomes) criteria [4]. Accordingly, we stopped antihypertensive medication and gentamicin infusions and adjusted the dosages of colistimethate and meropenem for renal function. Complete recovery of renal function occurred 7 days after gentamicin was discontinued. Because of the marked clinical improvement, we decided to continue administering meropenem and colistimethate at full dosages. Surprisingly, 2 weeks after starting therapy with colistimethate, we observed a gradual and sustained decline in serum potassium levels, as shown in Fig. 1, without any apparent cause of electrolyte loss. The hypokalaemia persisted despite daily intravenous supplementation of potassium chloride $30 \mathrm{mEq}$,

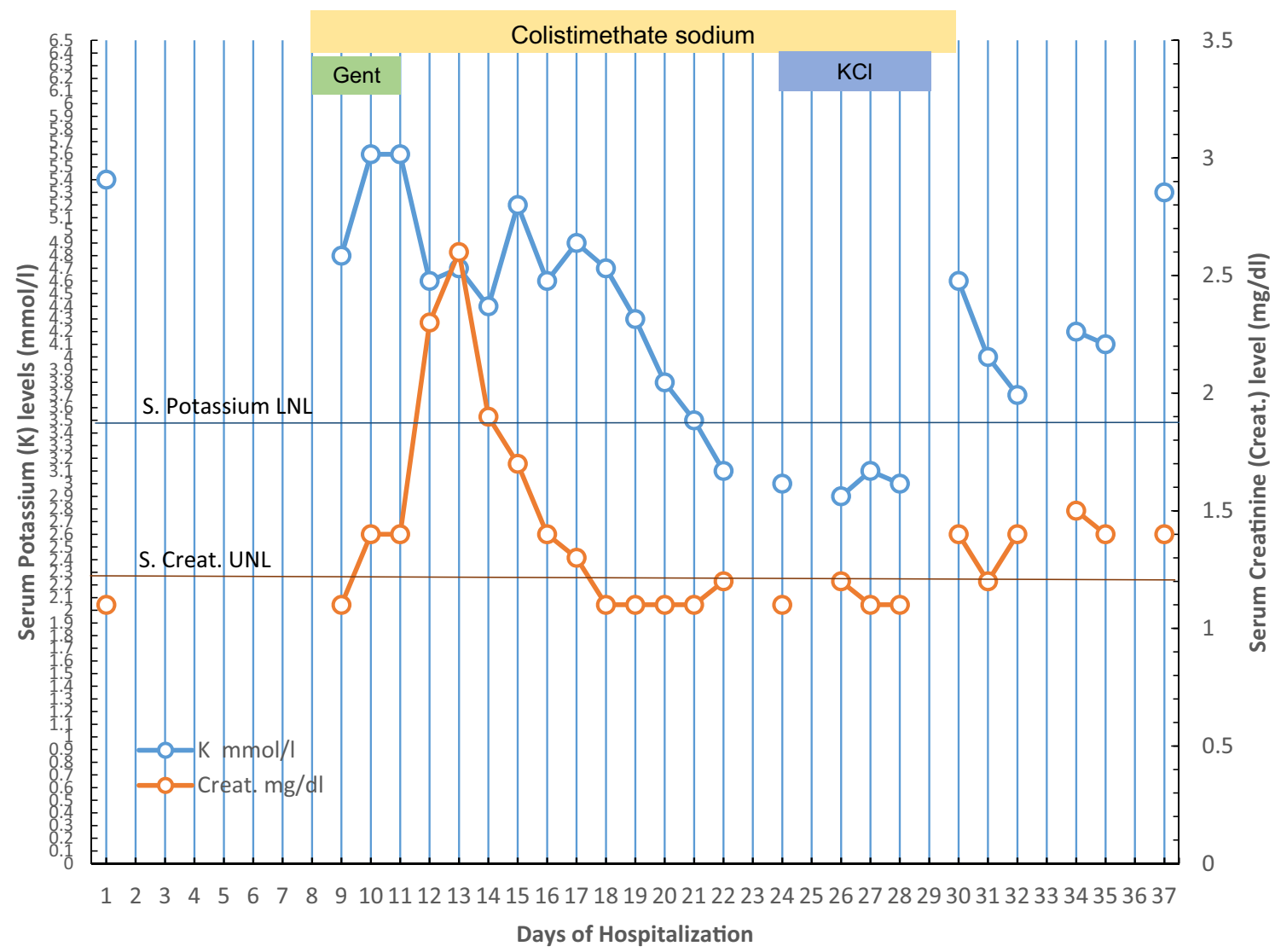

Fig. 1 Levels of serum potassium and creatinine followed and analysed throughout hospitalization of a case of ulcer infection receiving systemic colistimethate sodium. Lower normal limit (LNL) of serum potassium $(\mathrm{K})$ and upper normal limit (UNL) of serum creatinine (Creat.) are shown. Timing and duration of treatment with colistimethate sodium, gentamicin (Gent.) and potassium chloride supplementation $(\mathrm{KCl})$ is indicated 
reaching a nadir of $2.9 \mathrm{mmol} / \mathrm{l}$. The patient also exhibited mild hypocalcaemia, hypophosphataemia and hypomagnesaemia, whereas serum creatinine levels and urine output were normal. Repeated spot urinary electrolyte measurements showed a consistent urinary potassium excretion $>15 \mathrm{mmol} / \mathrm{l}$ with consistent urinary sodium $>100 \mathrm{mmol} / \mathrm{l}$. Urinary potassium excretion on a 24-h urine collection was $35 \mathrm{mmol} / 24 \mathrm{~h}$. Urinary cortisol levels were slightly below the normal range, whereas urinary calcium on a 24-h urine sample was within the normal range. Venous blood gas analysis repeatedly showed mild metabolic alkalosis $(\mathrm{pH}$ 7.47 and $\mathrm{HCO}_{3}{ }^{-} 34.9 \mathrm{mmol} / \mathrm{l}$ ). Moreover, the patient started complaining of lumbar pain. Renal ultrasound examination showed renal calyceal microlithiasis. Antibiotic treatment was discontinued after a total of 23 days. Serum electrolyte levels spontaneously returned to baseline levels 6 days after colistimethate administration was discontinued. The patient was discharged with low normal blood pressure levels and without any antihypertensive prescription.

\section{Discussion}

Our patient received prolonged therapy with colistimethate and developed unexplainable hypokalaemia, metabolic alkalosis and other electrolyte abnormalities of renal origin in course of therapy. The metabolic alterations, associated with the normalization of blood pressure levels in a hypertensive patient, and the development of microlithiasis, were all suggestive of Bartter syndrome, a group of closely related defects of the distal segment of the nephron. In Bartter syndrome, reabsorption of sodium chloride is impaired in the affected segment, leading to hypokalaemia, hypochloraemia and metabolic alkalosis. Some patients also present with hypercalciuria and nephrocalcinosis [5]. Classically, Bartter syndrome is congenital; however, authors have described acquired forms developing during treatment with different classes of drugs, including tuberculostatics and aminoglycosides [6].

Although our patient received therapy with other drugs, the reaction appeared only after treatment with colistimethate and disappeared after it was discontinued. This is not true for other potentially causative drugs such as the ACE inhibitor, which the patient took for a long time before admission without exhibiting any known alterations, and that was interrupted long before the reaction appeared. Similarly, the short duration of therapy with gentamicin and the onset of hypokalaemia long after its discontinuation makes it unlikely to be the cause. On the other hand, the onset of the metabolic alterations during treatment with colistimethate and resolution after interruption are suggestive of an association and possibly even a causative relationship despite the lack of rechallenge or pharmacokinetic/ pharmacodynamic studies. This is further supported by another case in the literature that reported the development of Bartter-like syndrome in a preterm infant [7]. However, to our knowledge, this is the first report in an adult.

\section{Conclusion}

Albeit limited to a single case, this report suggests that prolonged systemic therapy with colistimethate sodium may be associated with persistent refractory hypokalaemia and Bartter-like syndrome. Further research is needed to clearly define the phenomenon, especially in light of the increasing utilization of colistimethate even in patients in generally satisfactory condition.

\section{Compliance with ethical standards}

Funding No funds were received in support of this work.

Conflict of interest Tarek Kamal Eldin, Grazia Tosone, Alfredo Capuano and Raffaele Orlando have no conflicts of interest.

Ethics The patient provided written informed consent for publication of this case report. A copy of the written consent may be requested for review from the corresponding author.

Open Access This article is distributed under the terms of the Creative Commons Attribution-NonCommercial 4.0 International License (http://creativecommons.org/licenses/by-nc/4.0/), which permits any noncommercial use, distribution, and reproduction in any medium, provided you give appropriate credit to the original author(s) and the source, provide a link to the Creative Commons license, and indicate if changes were made.

\section{References}

1. Goodman L, Gilman A, Brunton L. Goodman and Gilman's manual of pharmacology and therapeutics. New York: McGrawHill Medical; 2011.

2. Spapen H, Jacobs R, Van Gorp V, Troubleyn J, Honoré PM. Renal and neurological side effects of colistin in critically ill patients. Ann Intensive Care. 2011;1:14.

3. Magiorakos A-P, Srinivasan A, Carey RB, Carmeli Y, Falagas ME, Giske CG, et al. Multidrug-resistant, extensively drugresistant and pandrug-resistant bacteria: an international expert proposal for interim standard definitions for acquired resistance. Clin Microbiol Infect. 2012;18(3):268-81.

4. Kellum J, Lameire N, Aspelin P, Barsoum RS, Burdmann E, Goldstein SL, et al. KDIGO Clinical Practice Guideline for Acute Kidney Injury. Kidney Int Suppl. 2012;2:1-138. http://www. pubmedcentral.nih.gov/articlerender.fcgi.

5. Kleta R, Bockenhauer D. Bartter syndromes and other salt-losing tubulopathies. Nephron Physiol. 2006;104(2):73-80. doi:10.1159/ 000094001.

6. Bartter and Gitelman syndromes. (2017). https://www.uptodate. com/contents/bartter-and-gitelman-syndromes. Accessed 8 Jul 2017.

7. Cakir U, Alan S, Zeybek C, Erdeve O, Atasay B, Yalcinkaya F, et al. Acquired bartter-like syndrome associated with colistin use in a preterm infant. Ren Fail. 2013;35(3):411-3. 